\title{
Pupils' Knowledge Level about the Contraindicationsof Cardiovascular Diseases of the Heart like Dilated Cardiomyopathy (DCM), Hypertensive Heart Disease (HHD) and Pulmonary Heart Disease (PHD) as Health Education Strategy in Preventive Health
}

\author{
Bebeley Samuel Joseph, Laggao Sam Augustine and Tucker Henry Joe
}

\begin{abstract}
Aim: The objective of this study was to point out pupils' knowledge level about the contraindications of cardiovascular diseases of the heart like Dilated Cardiomyopathy (DCM), Hypertensive Heart Disease (HHD) and Pulmonary Heart Disease (PHD) as health education strategy in preventive health cased at University Secondary School, Njala. Method: The researcher interviewed mainly high school pupils with a sampled number of one hundred and twenty $(n=120) .46 \%(n=55)$ female and 54\% $(n=65)$ male; $67 \%(n=80)$ Christians; $33 \%(\mathrm{n}=40)$ Muslims; were selected using the stratified random sampling. Also stratified were58\% ( $n=70)$ from the option Pure Science-to-General Science;42\% ( $n=50)$ from the option Social Science-to-Applied Art; 29.2\% ( $\mathrm{n}=35)$ from SSS(One-to-Two); $70.8 \%(\mathrm{n}=85)$ from SSS(Three-to-Four);62.8\% $(\mathrm{n}=75)$ within the age circle of (13-to$17) ; 37.5 \%(\mathrm{n}=45)$ within the age circle of $(18$-to-25+) in years. Results: The variables of the finding were tested@ level of significance $\mathrm{p}<0.05$ through a statistical instrument of T-test. Analysis from the study objective, shows negative skewedness in all three variables as placed in tabulated values (t-scores of-0.802, 0.529 and 2.129) of 2, 4 and 6 when tested@p < 0.05. Conclusion: In summing up due to the negative skewedness in all of the variables tested, the finding has demonstrated majority view of high school pupils' low level of knowledge about the contraindications of cardiovascular diseases of the heart as health education strategy in preventive health cased at University Secondary School.The researchers recommend that health education as a teaching subject be included in the schools' curriculum to help in the teaching of preventive health to school pupils.
\end{abstract}

\author{
Bebeley Samuel Joseph \\ $\mathrm{Ph}$.D. Scholar \\ Shanghai University of Sport, China \\ Email: bsaj2004@hotmail.co.uk ; bsaj2004@gmail.com \\ Laggao Sam Augustine \\ HOD \\ Human Kinetics and Health Education \\ Njala University, Sierra Leone \\ Tucker Henry Joe \\ Lecturer \\ Human Kinetics and Health Education \\ Njala University, Sierra Leone
}

\begin{abstract}
Key Words: Dilated Cardiomyopathy, Hypertensive, Heart Disease, Pulmonary Disease
\end{abstract}

DOI: $10.18376 / j e s p / 2017 / v 13 / \mathbf{i} 2 / 111278$

\section{Introduction}

Health Education according to World Health Organization (1998), is a composition of consciously constructed opportunities for learning involving some form of communication designed to improve health literacy, including improving knowledge, and developing life skills that are conducive to individual,community and environmental health. A disease is a particular abnormal condition, a disorder of a structure or function that affects part or all of an organism. The causal study of disease is called pathology. Disease is often construed as a medical condition associated with specific symptoms and signs, McWhinney, I. R. (1987). It may be caused by factors originally from an external source, such as infectious disease, or it may be caused by internal dysfunctions, such as 
autoimmune diseases, McWhinney, I. R. (1987).Infection on the other hand begins when an organism successfully colonizes by entering the body, growing and multiplying. Most humans are not easily infected. Those who are weak, sick, and malnourished, have cancer or are diabetic have increased susceptibility to chronic or persistent infections. Individuals who have a suppressed immune system are particularly susceptible to opportunistic infections, Reddy, M., et al. (2012). Cardiovascular disease however, includes coronary artery diseases (CAD) such as angina and myocardial infarction (heart attack), Shanthi, M. et al. (2011). Other cardiovascular diseases are angina-coronary artery diseases (CAD), myocardial infection, stroke, hypertensive heart disease, congenital heart disease, rheumatic heart disease, cardiomyopathy, atrial fibrillation, endocarditis, peripheral artery disease and venous thrombosis, Global Burden of Disease Study (2013).

Dilated cardiomyopathy (DCM) under study is a condition in which the heart becomes enlarged and cannot pumpblood efficiently. The decreased heart function can affect the lungs, liver, and other body systems, Jameson, J. N. et al. (2005). Dilated cardiomyopathy (DCM) is one of the cardiomyopathies, a group of diseases that affect primarily the myocardium (muscle of the heart). Different cardiomyopathies have different causes and affect the heart in different ways, Jameson, J. N. et al. (2005). In Dilated cardiomyopathy (DCM), a portion of the myocardium is dilated, often without any obvious cause. Left or right ventricular systolic pump function of the heart is impaired, leading to progressive cardiac enlargement and hypertrophy, a process called remodeling,Jameson, J. N. et al. (2005). Dilated cardiomyopathy is the most common form of non-ischemic cardiomyopathy. It occurs more frequently in men than in women, and is most common between the ages of 20 and 60 years, Robbins, S. L. et al. (2003). About one in three cases of congestive heart failure (CHF) is due to dilated cardiomyopathy, Jameson, J. N. et al. (2005). The progression of heart failure is associated with left ventricular remodeling, which manifests as gradual increases in left ventricular end-diastolic and end-systolic volumes, wall thinning, and a change in chamber geometry to a more spherical, less elongated shape, Pieske, B. (2004). This process is usually associated with a continuous decline in ejection fraction.

Hypertensive heart disease (HHD) on the other hand includes a number of complications of high blood pressure that affect the heart. While there are several definitions of hypertensive heart disease in the medical literature, Alegría-Ezquerra, E. et al. (2006) and Lip, G. Y. et al (2000), the term is most widely used in the context of the International Classification of Diseases (ICD) coding categories. The definition includes heart failure and other cardiac complications of hypertension when a causal relationship between the heart disease and hypertension is stated or implied on the death certificate, Lip, G. Y. et al (2000). However, since high blood pressure is a risk factor for atherosclerosis and ischemic heart disease, Grossman, E. et al. (1996), death rates from hypertensive heart disease provide an incomplete measure of the burden of disease due to high blood pressure, Grossman, E. et al. (1996), In 2013 hypertensive heart disease resulted in 1.07 million deaths up from 630,000 in 1990, Global Burden of Disease Study 2013 Mortality and Causes of Death, Collaborators (2014). Because there are no symptoms with high blood pressure, people can have the condition without knowing it. Diagnosing high blood pressure early can help prevent heart disease, stroke, eye problems, and chronic kidney disease, Maeder, M. T. et al. (2009). Hypertensive heart disease was estimated to be responsible for 1.0 million deaths worldwide in 2004 (or approximately 1.7\% of all deaths globally), and was ranked 13th in the leading global causes of death for all ages, World Health Organization (2008). A world map shows the estimated disability-adjusted life years per 100,000 inhabitants lost due to hypertensive heart disease in 2004, "WHO Disease and injury country estimates" (2009).

With Pulmonary Heart Disease (PHD), it is the enlargement and failure of the right ventricle of the heart as a response to increased vascular resistance or high blood pressure in the lungs (pulmonary hypertension), American Medical Network-Pulmonary Heart Disease (2010). Chronic pulmonary 
heart disease usually results in right ventricular hypertrophy (RVH), whereas acute pulmonary heart disease usually results in dilatation. Hypertrophy is an adaptive response to a long-term increase in pressure, American Medical Network-Pulmonary Heart Disease (2010). Individual muscle cells grow larger (in thickness) and change to drive the increased contractile force required in moving the blood against greater resistance. Dilatation is a stretching (in length) of the ventricle in response to acute increased pressure, such as when caused by a pulmonary embolism or ARDS (acute respiratory distress syndrome), American Medical Network-Pulmonary Heart Disease (2010). To be classified as pulmonary heart disease, the cause must originate in the pulmonary circulation system. Two major causes are vascular changes as a result of tissue damage (e.g. disease, hypoxic injury, chemical agents, etc.) and chronic hypoxic pulmonary vasoconstriction. If left untreated, then death may result. RVH due to a systemic defect is not classified as pulmonary heart disease, American Medical Network-Pulmonary Heart Disease (2010). The heart and lungs are intricately related. Whenever the heart is affected by disease, the lungs will follow and vice versa. Pulmonary heart disease is by definition a condition when the lungs cause the heart to fail, American Medical Network-Pulmonary Heart Disease (2010).

This study only points out onhigh school pupils' knowledge level about the contraindications of Cardiovascular Diseases of the Heart likeDilated Cardiomyopathy (DCM), Hypertensive Heart Disease (HHD) and Pulmonary Heart Disease (PHD), as health educationstrategy in preventive health, ranging from SSS One-to-Two and SSS Three-to-Fourcased at University Secondary School Njala, Southern Sierra Leone.

\section{Materials and Methods}

Selection of Participants: The researchers mainly interviewed high school pupils with asampled number of one hundred and twenty $(n=120)$, of which $46 \%(n=55)$ female and $54 \%(n=65)$ male; $67 \%(\mathrm{n}=80)$ Christians; 33\% ( $\mathrm{n}=40)$ Muslims; were selected using the stratified random sampling. Also stratified were $58 \%(n=70)$ from the option Pure Science-to-General Science; $42 \%(n=50)$ from the option Social Science-to-Applied Art; 29.2\% $(n=35)$ from SSS-One-to-Two; 70.8\% $(\mathrm{n}=85)$ from SSS-Three-to-Four; 62.8\% $(\mathrm{n}=75)$ within the age circle of $(13$-to-17); 37.5\% $(\mathrm{n}=45)$ within the age circle of (18-to-25+) in years.

Instrument for Measuring the Parameters: The survey questionnaire design was adopted for the study. The variables tested were knowledge about Dilated Cardiomyopathy (DCM), Hypertensive Heart Disease (HHD) and Pulmonary Heart Disease (PHD).The restructured but validated pupils' knowledge level about health education strategy in preventive healthquestionnaire (PKHEPHQ) was the adopted instrument for testing the parameters, formally utilized by Bebeley, (2016).Senior colleaguesfrom the School of Community and Clinical Health Sciencesand the School of Education, Njala University validated the questionnaire, which was pre-tested on sixty pupils $(\mathrm{n}=60)$ of Saint Andrews Senior Secondary School, BoSouthern Sierra Leone;with an intra-class correlation coefficient (ICC) reliability of0.89-to-0.99 using the ANOVA technique.

Test Procedures: The one hundred and twenty randomly selected using the stratified random samplingmethod of school pupils $(\mathrm{n}=120)$ mainly from senior forms at University Secondary School, Njala were interviewedon the School'spremisesby the researchers with the help of some academic staff members of Human Kinetics and Health Education Department, to respond to the "Yes-I-Do" or "No-I-Don't"variablesadopting the face to face technique. Therespondents'views were stratified accordingly for statistical analyses.

Statistical Analysis: The frequency distribution, percentage, mean, standard deviation andT-test werethe statistical instruments used to compute, analyze and to find out any significant differences in the data obtained from high school pupils' knowledge level about the contraindications ofcardiovascular diseases of the heart as health education strategy in preventive health. The results were tested @ level of significance $\mathrm{p}<0.05$. 
Results

Table 1. High/Senior School pupils' knowledge about Dilated Cardiomyopathy [n=120]

\begin{tabular}{|c|c|c|c|c|c|c|c|c|c|}
\hline No. & $\begin{array}{l}\text { Item-One: Dilated Cardiomyopathy } \\
\text { (DCM) }\end{array}$ & $\sqrt{a} \delta_{i}$ & $3^{9}$ & 死 & 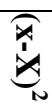 & \& 7 & $3^{9}$ & (4) & 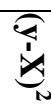 \\
\hline 1 & $\begin{array}{l}\text { Do you know that dilated } \\
\text { cardiomyopathy can be linked to } \\
\text { shortness of breath, syncope and angina } \\
\text { but only in the presence of ischemic heart } \\
\text { disease? }\end{array}$ & י & $\begin{array}{l}\vec{b} \\
\text { iv }\end{array}$ & $\underset{i}{\stackrel{+}{N}}$ & $\begin{array}{l}\vec{\sigma} \\
w \\
\omega \\
\dot{\phi}\end{array}$ & Nu & $\underset{\infty}{\mathbb{O}}$ & $\underset{+}{+}$ & $\begin{array}{l}\vec{\sigma} \\
w \\
\omega \\
\dot{\phi}\end{array}$ \\
\hline 2 & $\begin{array}{l}\text { Do you know that a person suffering } \\
\text { from dilated cardiomyopathy may have } \\
\text { an enlarged heart, with pulmonary edema } \\
\text { (a condition characterized by an excess } \\
\text { of watery fluid collecting in the cavities } \\
\text { or tissues of the body) and an elevated } \\
\text { jugular (neck or throat) venous pressure } \\
\text { and a low pulse pressure? }\end{array}$ & $\vec{u}$ & $\begin{array}{l}\mathrm{N} \\
\mathrm{in}\end{array}$ & $\stackrel{N}{+}$ & $\begin{array}{l}4 \\
\dot{\infty} \\
\ddot{1} \\
\dot{\phi}\end{array}$ & $\vec{\Delta}$ & $\begin{array}{l}w \\
\text { ir }\end{array}$ & 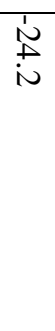 & $\begin{array}{l}\text { y } \\
\infty \\
\dot{\phi} \\
\dot{\phi}\end{array}$ \\
\hline 3 & $\begin{array}{l}\text { Do you know that dilated } \\
\text { cardiomyopathy is probably the result of } \\
\text { damage to the myocardium produced by } \\
\text { a variety of toxic, metabolic, or } \\
\text { infectious agents? }\end{array}$ & $\dot{v}$ & $\tilde{u}_{i}$ & $\begin{array}{l}\dot{\prime} \\
\dot{\infty}\end{array}$ & $\begin{array}{l}w \\
\dot{\phi} \\
\dot{\phi}\end{array}$ & $\bar{u}$ & $\stackrel{N}{N}$ & $\ddot{\infty}$ & $\begin{array}{l}w \\
\dot{\phi} \\
\dot{\phi}\end{array}$ \\
\hline 4 & $\begin{array}{l}\text { Do you know that dilated } \\
\text { cardiomyopathy may be as a result of late } \\
\text { sequelae (condition of a previous disease } \\
\text { or injury) of acute viral myocarditis, such } \\
\text { as with Coxsackie B virus (enterovirus } \\
\text { causing various respiratory, neurological } \\
\text { and muscular diseases) and other } \\
\text { enteroviruses (causing polio and } \\
\text { hepatitis A which occur chiefly in the } \\
\text { gastrointestinal track)? }\end{array}$ & Nu & $\underset{\infty}{\infty}$ & $\begin{array}{l}\text { Nu } \\
\stackrel{\sim}{\prime}\end{array}$ & $\begin{array}{l}\text { gे } \\
\dot{1} \\
\dot{1}\end{array}$ & o & $\begin{array}{l}\text { তै } \\
\text { i }\end{array}$ & $\begin{array}{l}\stackrel{N}{u} \\
\infty\end{array}$ & $\begin{array}{l}2 \\
\dot{a} \\
\dot{+}\end{array}$ \\
\hline 5 & $\begin{array}{l}\text { Do you know that autoimmune (denoting } \\
\text { disease caused by antibodies or } \\
\text { lymphocytes produced against substances } \\
\text { naturally present in the body) } \\
\text { mechanisms are also suggested as a cause } \\
\text { for dilated cardiomyopathy? }\end{array}$ & $\breve{o}$ & $\mathrm{~N}$ & $\underset{\infty}{\stackrel{1}{\infty}}$ & $\begin{array}{l}\stackrel{\oplus}{w} \\
\stackrel{N}{\phi} \\
\dot{\phi}\end{array}$ & 8 & $\vec{u}$ & $\underset{\infty}{\mathbb{O}}$ & 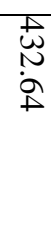 \\
\hline 6 & $\begin{array}{l}\text { Do you know that individuals subjected } \\
\text { with an extremely high occurrence of } \\
\text { premature ventricular contractions ( } a \\
\text { heartbeat outside the normal rhythm) can } \\
\text { develop dilated cardiomyopathy? }\end{array}$ & $w_{u}$ & $\begin{array}{l}\text { vo } \\
\text { iv }\end{array}$ & $\begin{array}{l}\vec{r} \\
\dot{\infty}\end{array}$ & 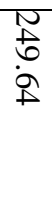 & $\infty$ & $\underset{\infty}{\grave{\infty}}$ & $\vec{\infty}$ & 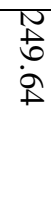 \\
\hline
\end{tabular}


Journal of Exercise Science \& Physiotherapy, Vol. 13, No. 2, 2017

ISSN: 0973-2020 (Print) I I OR Impact Factor = 5.23 UGC Approved [Journal No.7485] ISSN: 2454-6089 (online)

\begin{tabular}{|c|c|c|c|c|c|}
\hline II & $\begin{array}{l}\text { Yes }(x)[\text { Mean }=50.8 \& \text { SD }=25.6] \\
\text { No }(y)[\text { Mean }=69.2 \& \text { SD }=25.6]\end{array}$ & 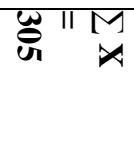 & 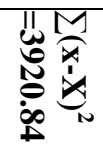 & 党 $\| M$ & 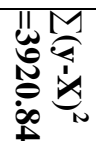 \\
\hline
\end{tabular}

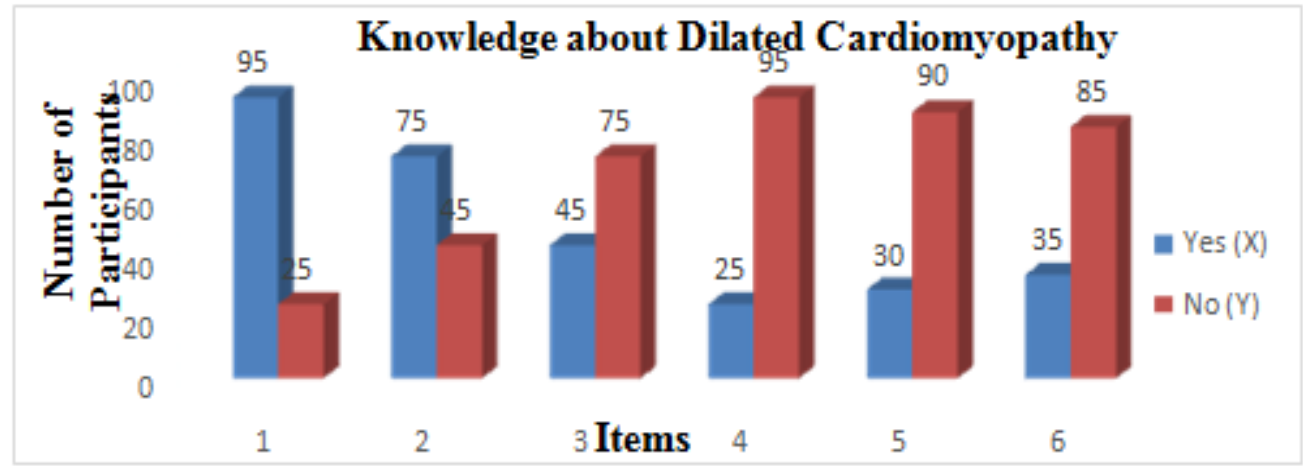

Figure 1. A chart showing pupils' knowledge about Dilated Cardiomyopathy[n=120]

Table 2. Dependent t-test ( $t$ ) analysis of pupils' knowledge about Dilated Cardiomyopathy $[\mathbf{n}=\mathbf{1 2 0}]$

\begin{tabular}{|c|c|c|c|c|}
\hline \multirow[t]{2}{*}{ Items } & \multicolumn{2}{|c|}{ Dilated Cardiomyopathy (DCM) } & \multirow[t]{2}{*}{$\mathrm{D}(\mathrm{X}-\mathrm{Y})$} & \multirow[t]{2}{*}{$\mathrm{D}^{2}$} \\
\hline & Yes $(\mathrm{X})$ & No $(Y)$ & & \\
\hline 1 & 95 & 25 & 70 & 4900 \\
\hline 2 & 75 & 45 & 30 & 900 \\
\hline 3 & 45 & 75 & -30 & 900 \\
\hline 4 & 25 & 95 & -70 & 4900 \\
\hline 5 & 30 & 90 & -60 & 3600 \\
\hline 6 & 35 & 85 & -50 & 2500 \\
\hline \multirow[b]{2}{*}{$*(n=6)$} & \multirow[b]{2}{*}{$*\left(\sum D\right)^{2}=12100$} & \multirow[b]{2}{*}{$* \mathbf{d f}=\mathbf{5}$} & $* \sum \mathbf{D}=\mathbf{- 1 1 0}$ & $* \sum D^{2}=17700$ \\
\hline & & & $* t=-\mathbf{0 . 8 0 2}$ & \\
\hline
\end{tabular}

Table 3. High/Senior School pupils' knowledge about Hypertensive Heart Disease [n=120]

\begin{tabular}{|c|c|c|c|c|c|c|c|c|c|}
\hline No. & $\begin{array}{l}\text { Item-Two: Hypertensive } \quad \text { Heart } \\
\text { Disease (HHD) }\end{array}$ & $(\widehat{x} \delta$ & $0^{9}$ & $\underset{y}{x}$ & 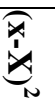 & (2) Z & $0^{9}$ & 㐫 & 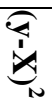 \\
\hline 1 & $\begin{array}{l}\text { Do you know that hypertensive heart } \\
\text { disease can be linked to enlargement of } \\
\text { the heart (cardiomegaly) and heart } \\
\text { failure? }\end{array}$ & 8 & $\vec{u}$ & $\frac{1}{c}$ & 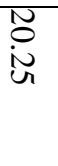 & $\breve{o}_{0}$ & $\stackrel{N}{u}$ & $\ddot{\dot{c}}$ & 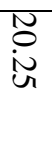 \\
\hline 2 & $\begin{array}{l}\text { Do you know that hypertensive heart } \\
\text { disease can also be linked to irregular } \\
\text { pulse or palpations and even fatigue? }\end{array}$ & $\overline{8}$ & $\underset{\omega}{\infty}$ & $\ddot{u}$ & 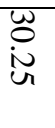 & Tु & $\vec{a}$ & $\begin{array}{l}\dot{u} \\
\text { ur }\end{array}$ & $\begin{array}{l}\mathscr{P}_{0} \\
\text { in }\end{array}$ \\
\hline
\end{tabular}




\begin{tabular}{|c|c|c|c|c|c|c|c|c|c|}
\hline 3 & $\begin{array}{l}\text { Do you know that hypertensive heart } \\
\text { disease can be also linked to weight gain } \\
\text { and swelling of the foot and ankles? }\end{array}$ & $\vec{\Xi}$ & $\stackrel{0}{v}$ & $\overline{u r}_{i}$ & $\begin{array}{l}N \\
\dot{1} \\
\text { N } \\
\text { Un }\end{array}$ & $\overline{0}$ & $\omega_{\omega}^{\infty}$ & $\begin{array}{l}\frac{1}{u r} \\
\dot{u}\end{array}$ & $\begin{array}{l}\text { N } \\
\text { D } \\
\text { N } \\
\text { Un }\end{array}$ \\
\hline 4 & $\begin{array}{l}\text { Do you know that hypertensive heart } \\
\text { disease can be linked to shortness of } \\
\text { breath and difficulty in sleeping flat in } \\
\text { bed (orthopnea)? }\end{array}$ & $\overrightarrow{c_{r}}$ & $\stackrel{\infty}{i}_{i}^{\infty}$ & $\vec{i}$ & $\begin{array}{l}\text { F } \\
\text { i } \\
\text { úr }\end{array}$ & $\vec{u}$ & $\bar{N}$ & $\stackrel{1}{\circ}$ & $\begin{array}{l}\text { F } \\
\text { ì } \\
\text { un }\end{array}$ \\
\hline 5 & $\begin{array}{l}\text { Do you know that hypertensive heart } \\
\text { disease can be linked to Bloating } \\
\text { (conditioned by an accumulation of gas } \\
\text { in the stomach) and abdominal pain and } \\
\text { greater need to urinate at night? }\end{array}$ & जे & $\stackrel{w}{+}$ & $\begin{array}{l}\text { N } \\
\text { in }\end{array}$ & 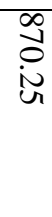 & $\tilde{u}_{1}$ & $\underset{\infty}{\stackrel{+}{c}}$ & $\begin{array}{l}\text { No } \\
\text { ir }\end{array}$ & $\begin{array}{l}\infty \\
\substack{0 \\
\sim} \\
\text { un }\end{array}$ \\
\hline 6 & $\begin{array}{l}\text { Do you know that people with } \\
\text { hypertensive heart disease suffers from } \\
\text { acutely heart failure and pulmonary } \\
\text { edema (conditioned by an excess watery } \\
\text { fluid collecting in the cavities or tissues } \\
\text { of the body) due to sudden failure of } \\
\text { pump function of the heart? }\end{array}$ & 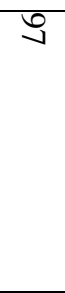 & $\stackrel{\infty}{\infty}_{\infty}^{\infty}$ & $\mathcal{N}_{n}$ & $\begin{array}{l}a \\
\text { úr }\end{array}$ & 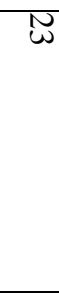 & $\begin{array}{l}\vec{b} \\
\text { i }\end{array}$ & ì & $\stackrel{i}{u}$ \\
\hline$\widehat{\underline{\Xi}}$ & $\begin{array}{l}\text { Yes }(x)[\text { Mean }=94.5 \& \text { SD }=14.6] \\
\text { No }(y)[\text { Mean }=25.5 \& \text { SD }=14.6]\end{array}$ & प̆ & & & $\underbrace{1}_{\substack{x \\
⿱ 亠 凶}}$ & $\overline{u_{\omega}}$ & $M$ & & 崔 \\
\hline
\end{tabular}

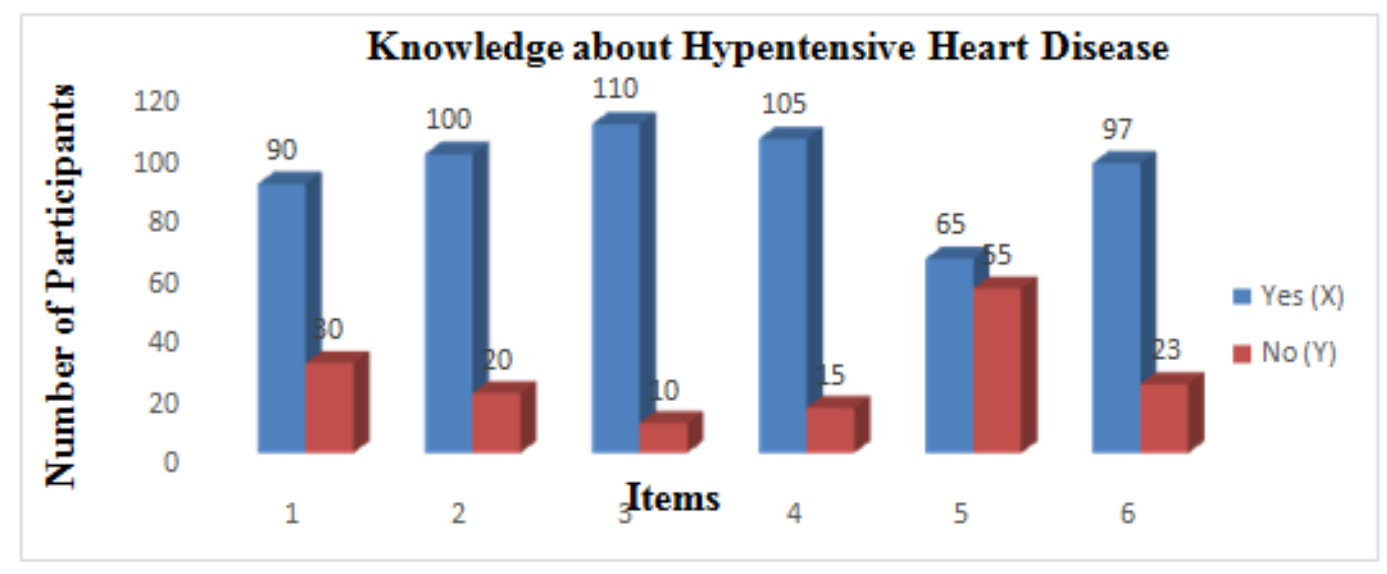

Figure 2. A chart showing pupils' knowledge about Hypertensive Heart Disease [n=120]

Table 4: Dependent t-test ( $t$ ) analysis of pupils' knowledge about Hypertensive Heart

Disease[n=120] 
Journal of Exercise Science \& Physiotherapy, Vol. 13, No. 2, 2017

ISSN: 0973-2020 (Print) I I OR Impact Factor = 5.23 UGC Approved [Journal No.7485] ISSN: 2454-6089 (online)

\begin{tabular}{|c|c|c|c|c|}
\hline \multirow[t]{2}{*}{ Items } & \multicolumn{2}{|c|}{$\begin{array}{l}\text { Hypertensive } \\
\text { (HHD) }\end{array}$} & \multirow[t]{2}{*}{$\begin{array}{ll} & \text { D } \\
\text { Y }\end{array}$} & \multirow[t]{2}{*}{$D^{2}$} \\
\hline & Yes $(X)$ & No $(\mathbf{Y})$ & & \\
\hline 1 & 90 & 30 & 60 & 3600 \\
\hline 2 & 100 & 20 & 80 & 6400 \\
\hline 3 & 110 & 10 & 100 & 10000 \\
\hline 4 & 105 & 15 & 90 & 8100 \\
\hline 5 & 65 & 55 & 10 & 100 \\
\hline 6 & 97 & 23 & 74 & 5476 \\
\hline \multirow[b]{2}{*}{$*(n=6)$} & \multirow{2}{*}{\multicolumn{2}{|c|}{$*\left(\sum D\right)^{2}=171396$}} & $* \sum D=414$ & $* \sum D^{2}=33676$ \\
\hline & & & $* \mathrm{t}=0.529$ & .571 \\
\hline
\end{tabular}

Table 5: High/Senior School pupils' knowledge about Pulmonary Heart Disease[n=120]

\begin{tabular}{|c|c|c|c|c|c|c|c|c|c|}
\hline No. & $\begin{array}{l}\text { Item-Three: Pulmonary Heart Disease } \\
\text { (PHD) }\end{array}$ & $\widehat{\star 凶} \approx$ & $3^{9}$ & 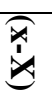 & 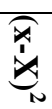 & e Z & $d^{9}$ & $\dot{4}$ & 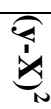 \\
\hline 1 & $\begin{array}{l}\text { Do you know that pulmonary heart } \\
\text { disease can be linked to Shortness of } \\
\text { breath which occurs on exertion but } \\
\text { when severe can occur at rest? }\end{array}$ & ত্ & $\begin{array}{l}\mathscr{1} \\
\dot{w}\end{array}$ & $\stackrel{1}{\prime}$ & 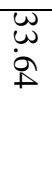 & y̆ & $\stackrel{\oplus}{*}$ & $\stackrel{c}{\infty}$ & $\begin{array}{l}w \\
\dot{\phi} \\
\dot{\phi}\end{array}$ \\
\hline 2 & $\begin{array}{l}\text { Do you know that pulmonary heart } \\
\text { disease can be linked to wheeze } \\
\text { (whistling or ratting breath sound in the } \\
\text { chest, due to obstruction in the air } \\
\text { passage) and chronic wet cough? }\end{array}$ & ur & $\begin{array}{l}\stackrel{\Delta}{\longrightarrow} \\
\infty\end{array}$ & $\begin{array}{l}\stackrel{1}{0} \\
\infty \\
\infty\end{array}$ & $\begin{array}{l}\stackrel{+}{\omega} \\
\stackrel{N}{a} \\
\stackrel{\perp}{\perp}\end{array}$ & जे & $\stackrel{w}{\stackrel{H}{N}}$ & $\underset{\infty}{\mathbb{\infty}}$ & $\begin{array}{l}\stackrel{+}{\omega} \\
\stackrel{N}{a} \\
\dot{\phi}\end{array}$ \\
\hline 3 & $\begin{array}{l}\text { Do you that pulmonary heart disease can } \\
\text { be linked to swelling of the abdomen } \\
\text { with fluid (ascites) and swelling of the } \\
\text { ankles and feet (pedal edema)? }\end{array}$ & 9 & $\breve{o}_{\infty}$ & $\underset{\infty}{+\infty}$ & $\begin{array}{l}\text { No } \\
\dot{0} \\
\dot{\phi}\end{array}$ & पु & $\begin{array}{l}\vec{t} \\
\dot{N}\end{array}$ & $\underset{\infty}{\vec{\phi}}$ & 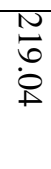 \\
\hline 4 & $\begin{array}{l}\text { Do you know that pulmonary heart } \\
\text { disease can be linked to enlargement or } \\
\text { prominent neck and facial veins and } \\
\text { raised jugular venous pressure (JVP i.e. } \\
\text { pressure in any of the large veins in the } \\
\text { neck, carrying blood from the head)? }\end{array}$ & $\vec{\omega}$ & 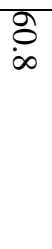 & $\stackrel{N}{\infty}$ & $\stackrel{+}{\infty}$ & $\stackrel{\oplus}{\forall}$ & $\begin{array}{l}\text { w } \\
\text { iv }\end{array}$ & $\stackrel{N}{N}$ & $\underset{\phi}{\infty}$ \\
\hline 5 & $\begin{array}{l}\text { Do you know that pulmonary heart } \\
\text { disease can be linked to enlargement of } \\
\text { the liver and bluish discoloration of the } \\
\text { skin (cyanosis)? }\end{array}$ & $\overline{8}$ & $\underset{\omega}{\infty}$ & $\stackrel{N}{\mathbb{N}}$ & $\begin{array}{l}\mathscr{y} \\
\infty \\
0 \\
\dot{\phi}\end{array}$ & $\widetilde{O}$ & $\vec{a}$ & 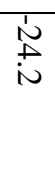 & $\begin{array}{l}\text { y } \\
\infty \\
\dot{1}\end{array}$ \\
\hline 6 & $\begin{array}{l}\text { Do you know that pulmonary heart } \\
\text { disease can be linked to the presence of } \\
\text { abnormal heart sounds and possible bi- } \\
\text { phasic atrial response shown on an EKG } \\
\text { due to hypertrophy (enlargement of an } \\
\text { organ or tissue resulting from an } \\
\text { increase in size of its cells)? }\end{array}$ & 2 & $\infty$ & O্ & $\begin{array}{l}+ \\
\stackrel{1}{0} \\
\infty \\
\dot{\phi}\end{array}$ & $\stackrel{N}{A}$ & No & ì & $\begin{array}{l}+ \\
\infty \\
\infty \\
+ \\
1\end{array}$ \\
\hline
\end{tabular}




\begin{tabular}{|c|c|c|c|c|c|}
\hline ఏ્త & $\begin{array}{l}\text { Yes }(x)[\text { Mean }=75.8 \& \text { SD }=16.8] \\
\text { No }(y)[\text { Mean }=44.2 \& \text { SD }=16.8]\end{array}$ & 岳 & 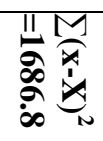 & 会"M & 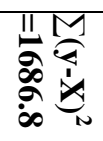 \\
\hline
\end{tabular}

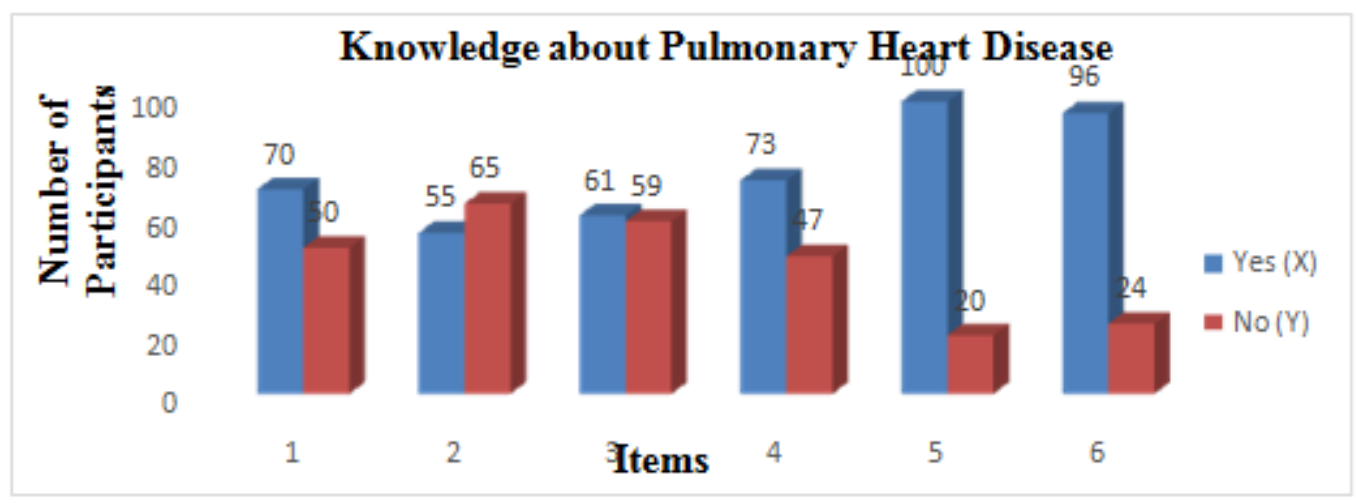

Figure 3: A chart showing pupils' knowledge about Pulmonary Heart Disease [n=120]

Table 6: Dependent t-test (t) analysis of pupils' knowledge about Pulmonary Heart

Disease[n=120]

\begin{tabular}{|l|l|l|l|l|}
\hline \multirow{2}{*}{ Items } & \multicolumn{2}{|l|}{ Pulmonary Heart Disease (PHD) } & \multirow{2}{*}{ D (X-Y) } & \multirow{2}{*}{$\mathbf{D}^{\mathbf{2}}$} \\
\cline { 2 - 5 } & Yes $(\mathbf{X})$ & No $(\mathbf{Y})$ & 20 & 400 \\
\hline 1 & 70 & 50 & -10 & 100 \\
\hline 2 & 55 & 65 & 02 & 04 \\
\hline 3 & 61 & 59 & 26 & 676 \\
\hline 4 & 73 & 47 & 80 & 6400 \\
\hline 5 & 100 & 20 & 72 & 5184 \\
\hline $\mathbf{6}$ & 96 & 24 & $* \sum \mathbf{D}=\mathbf{1 9 0}$ & $* \sum \mathbf{D}^{\mathbf{2}}=\mathbf{1 2 7 6 4}$ \\
\hline \multirow{2}{*}{$*(\mathbf{n}=\mathbf{6})$} & $*\left(\sum \mathbf{D}\right) \mathbf{~}^{\mathbf{2}}=\mathbf{3 6 1 0 0}$ & $* \mathbf{d f}=\mathbf{5}$ & $* \mathbf{2 . 1 2 9}$ & $* \mathbf{2 . 5 7 1}$ \\
\hline
\end{tabular}

\section{Discussion of Results}

While not all lung diseases can be prevented, one can reduce the risk of lung disease according to a published report by Living with Pulmonary Heart Disease (2010), through a means of avoiding or discontinuing smoking. Patients with chronic obstructive lung disease always end up with right heart failure. This study mainly focuses on pupils' knowledge level about the contraindications of cardiovascular diseases of the heart likeDilated Cardiomyopathy (DCM), Hypertensive Heart Disease (HHD) and Pulmonary Heart Disease (PHD), ashealth education strategy in preventive health amongst University Secondary School pupils cased at Njala, Sierra Leone. The frequency distribution, percentage $(\%)$, mean, standard deviation and Dependent t-test (t) were the statistical instruments used to compute and compare the results of the finding. The t-test results were tested $@ \mathrm{p}<0.05$ level of significance. In discussion, the result shows complete in significant differences ( $\mathrm{t}$-values $=-0.802,0.529$ and2.129) with regards high school pupils' knowledge level about the contraindications of cardiovascular diseases of the heart as health education strategy in preventive 
health. The finding clearly points out the need for the introduction of Health Education as a teaching subject in schools in other to help disseminate through teaching and sensitizations, the contraindications associated with cardiovascular diseases with respect to dilated cardiomyopathy which has the potential of affecting many individuals even to death of which school pupils are no exception as recorded in the insignificant difference in table2(t-value $=-0.802)$. In conformity, Mitchell, R. S. et al. (2003), reported in their publication that about 25-35\% of affected individuals with dilated cardiomyopathy (DCM) have familial forms of the disease, with most mutations affecting genes encoding cytoskeletal proteins, while according to Ross, J. (2002), some affect other proteins involved in contraction. However, death as reported by Pieske, B. (2004), is due to either congestive heart failure or bradycardia (abnormally slow heart action). Educating school pupils about modifying their lifestyle towards physical health will help greatly in preventing and or reducing cardiovascular diseases of the heart as reported by Chobanian, A. V. et al. (2003), that the risk of cardiovascular disease and death could be reduced by lifestyle modifications, including dietary advice, promotion of weight loss and regular aerobic exercise, moderation of alcohol intake and cessation of smoking. However, drug treatment according to Hunt, S. A. et al. (2009), may also be needed to control the hypertension and reduce the risk of cardiovascular disease and manage the heart failure, or as reported by Fuster, V. et al. (2011), can control cardiac arrhythmias. Looking also to educating pupils about environmental health will help eliminate or reduce drastically the contraindications associated cardiovascular diseases of the heart as reported by a published article on Living with Pulmonary Heart Disease (2010), that individuals working in environments where there are chemicals should wear masks to prevent inhalation of dust particles. In conclusion, the published report by Living with Pulmonary Heart Disease (2010) stated that elimination of the cause is the most important intervention, i.e. smoking must be stopped, along with exposure to second-hand smoke. Also exposure to dust, flames, house hold smoke and to cold weather are to be avoided. Finally, the findings are also displayed according to responses of the respondents to the "Yes-I-Do" or "No-I-Don't interview in a frequency distribution, percentages, mean and standard deviation values as indicated in tables 1, 3and5. In tables 1,3and5 holistically, greater number of respondents had little or no knowledge about the contraindications of cardiovascular diseases of the heart as health education strategy in preventive health.

Based on the results, it is therefore recommended that health education as a subject be included in the teaching curriculum in high schools for the teaching of physical health, environmental health and emotional and psychological healthas a strategy in preventive health with special reference to the contraindications ofcardiovascular diseases of the heart likeDilated Cardiomyopathy (DCM), Hypertensive Heart Disease (HHD) and Pulmonary Heart Disease (PHD), of which University Secondary School Njala is no exception.

\section{Conclusion}

This study was conducted purposely with the focus of pointing out facts about high school pupils' knowledge level about the contraindications ofcardiovascular diseases of the heart likeDilated Cardiomyopathy (DCM), Hypertensive Heart Disease (HHD) and Pulmonary Heart Disease (PHD),as health education strategy in preventive health with the aim of giving appropriate recommendations thereafter. Based on the findings, it is concluded that a good number of respondents had little or no knowledge about the contra indications associated with cardiovascular diseases of the heart as health education strategy in preventive health as displayed in their frequency distribution, percentage responses, mean scores and the calculated t-values tested@p $<$ 0.05 .

\section{Acknowledgement}

The authors express sincere thanks and appreciation to all staff and pupils of University Secondary School, Njala, whose immense co-operation rendered this study to fruition. 


\section{References}

Alegría-Ezquerra, E., González-Juanatey, J. R. and González-Maqueda, I. (2006): "Hypertensive heart disease: a proposed clinical classification". Revista Española de Cardiología (in Spanish): 59 (4): 398-9.

Allison, M. A., Denenberg, J. O. and Criqui, M. H. (2011): "Family History of Peripheral Artery Disease Is Associated with Prevalence and Severity of Peripheral Artery Disease". Journal of the American College of Cardiology:58 (13): 1386-92.

American Heart Association and American College of Sports Medicine: Joint Position Statement: Exercise and acute cardiovascular events: placing the risks into perspective. Med. Sci. Sports Exerc: 39:886-897, 2007.

American Medical Network-Pulmonary Heart Disease, Retrieved (2010).

Andreassi, J. L. (2000): Psychophysiology: human behavior and physiological response. Mahwah NJ: L. Erlbaum p. 287.

Artero, E. and España-Romero, V.et al. (2010): Health-related fitness in adolescents: underweight, and not only overweight, as an influencing factor. The AVENA study: Scand J Med SciSpor: 20 (3): 418427.

Barker, P. (2003): Psychiatric and Mental Health Nursing: The Craft of Caring. Great Britain: Arnold: ISBN 0340810262.

Bebeley, S. J. et al. (2016): Athletes' Knowledge about Preventing Sports Injuries like: Achilles Tendinitis, Runner's Knee/Patellofemoral Pain Syndrome and Shin Splints, as Prime Prevention Strategies in Slowing Ageing Process. Journal of Exercise Science and Physiotherapy: 12 (1): 22-28.

Boden, W. E., Franklin, B. and Berra, K.et al. (2014): "Exercise as a therapeutic intervention in patients with stable ischemic heart disease: an underfilled prescription." The American Journal of Medicine:127 (10): 905-11.

"Carotid Artery Stenosis information internal carotis occlusion Patient" Patient: Retrieved (2015).

"Cerebrovascular disease - Introduction - NHS Choices" www.nhs.uk: Retrieved (2015).

Chobanian, A. V. and Bakris, G. L.et al. (2003): National Heart, Lung, and Blood Institute Joint National Committee on Prevention, Detection, Evaluation, and Treatment of High Blood, Pressure, National High Blood Pressure Education Program Coordinating Committee "The Seventh Report of the Joint National Committee on Prevention, Detection, Evaluation, and Treatment of High Blood Pressure: the JNC 7 report" (PDF). JAMA:289 (19): 2560-2572.

Creager, M. A., Lüscher, T. F., Cosentino, F. and Beckman, J. A. (2003): "Diabetes and vascular disease pathophysiology, clinical consequences, and medical therapy: part I.".Circulation:108 (12): 15271532.

Deb, S., Wijeysundera, H. C., Ko, D. T., Tsubota, H., Hill, S. and Fremes, S. E. (2013): "Coronary artery bypass graft surgery vs percutaneous interventions in coronary revascularization: a systematic review." JAMA:310 (19): 2086-95.

Donatelle, R. (2009): Promoting Healthy Behavior Change. Health: The basics: (pp. 4). $8^{\text {th }}$ Ed. San Francisco, CA: Pearson Education, Inc.

Emson, H. E. (1987): "Health, disease and illness: matters for definition". CMAJ136 (8):

Fokkenrood, H. J., Bendermacher, B. L., Lauret, G. J., Willigendael, E. M., Prins, M. H. and Teijink, J. A. (2013): "Supervised exercise therapy versus non-supervised exercise therapy for intermittent claudication." The Cochrane database of systematic reviews8: CD005263.

Fowkes, F. G., Rudan, D. and Rudan, I.et al. (2013): "Comparison of global estimates of prevalence and risk factors for peripheral artery disease in 2000 and 2010: a systematic review and analysis." Lancet:382 (9901): 1329-40.

Fuster, V. andRydén, L. E. et al. (2011): "ACCF/AHA/HRS focused updates incorporated into the ACC/AHA/ESC 2006 Guidelines for the management of patients with atrial fibrillation: a report of the American College of Cardiology Foundation/American Heart Association Task Force on Practice Guidelines developed in partnership with the European Society of Cardiology and in collaboration with the European Heart Rhythm Association and the Heart Rhythm Society". Journal of the American College of Cardiology57 (11): e101-98.

GBD 2013 Mortality and Causes of Death, Collaborators (2014): "Global, regional and national age-sex specific all-cause and cause-specific mortality for 240 causes of death, 1990-2013: a systematic 


\section{Journal of Exercise Science \& Physiotherapy, Vol. 13, No. 2, 2017 \\ ISSN: $0973-2020$ (Print) $\quad \mathrm{I}_{2}$ OR Impact Factor = 5.23 UGC Approved [Journal No.7485] ISSN: 2454-6089 (online)}

analysis for the Global Burden of Disease Study 2013." Lancet: 385 (9963): 117-71.

Grossman, E. and Messerli, F. H. (1996): "Diabetic and hypertensive heart disease". Annals of Internal Medicine:125 (4): 304-310.

Gull, Sir William Withey (1894): T. D. Acland, ed. Medical Papers: p. 309.

Hankey, G. J., Norman, P. E. and Eikelboom, J. W. (2006): "Medical treatment of peripheral arterial disease." JAMA:295 (5): 547-53.

Herpertz-Dahlmann, B., Bühren, K. and Remschmidt, H. (2013): "Growing up is hard: Mental disorders in adolescence". DeutschesArzteblatt International:110 (25): 432-9; quiz 440.

Hunt, S. A. and Abraham, W. T.et al. (2009): "2009 focused update incorporated into the ACC/AHA 2005 Guidelines for the Diagnosis and Management of Heart Failure in Adults: a report of the American College of Cardiology Foundation/American Heart Association Task Force on Practice Guidelines: developed in collaboration with the International Society for Heart and Lung Transplantation". Circulation 119 (14): e391-479.

Hooi, J. D., Kester, A. D., Stoffers, H. E., Overdijk, M. M., van Ree, J. W. and Knottnerus, J. A. (2001): "Incidence of and risk factors for asymptomatic peripheral arterial occlusive disease: a longitudinal study". American Journal of Epidemiology:153 (7): 666-672.

Jameson, J. N., Kasper, D. L., Harrison, T. R., Braunwald, E., Fauci, A. S., Hauser, S. L. and Longo, D. L. (2005): Harrison's principles of internal medicine (16th ed.). New York: McGraw-Hill Medical Publishing Division: ISBN 0-07-140235-7.

Johnson, R. (2002): "The concept of sickness behavior: a brief chronological account of four key discoveries". Veterinary Immunology and Immunopathology87 (3-4): 443-450.

Joint Committee on Terminology (2001): "Report of the 2000 Joint Committee on Health Education and Promotion Terminology". American Journal of Health Education:32 (2): 89-103.

Joosten, M. M., Pai, J. K., Bertoia, M. L., Rimm, E. B., Spiegelman, D., Mittleman, M. A. and Mukamal, K. J. (2012): "Associations between conventional cardiovascular risk factors and risk of peripheral artery disease in men". JAMA:308 (16): 1660-7.

Kannel, W. B. and McGee, D. (1979): "Diabetes and glucose tolerance as risk factors for cardiovascular disease: The Framingham study". Diabetes Care:2 (2): 120-126.

Kwak, S. M., Myung, S. K., Lee, Y. J. and Seo, H. G. (2012): "Efficacy of Omega-3 Fatty Acid Supplements (Eicosapentaenoic Acid and Docosahexaenoic Acid) in the Secondary Prevention of Cardiovascular Disease: A Meta-analysis of Randomized, Double blind, Placebo-Controlled Trials". Archives of Internal Medicine:172 (9): 686-94.

Lip, G. Y., Felmeden, D. C., Li-Saw-Hee, F. L. and Beevers, D. G. (2000): "Hypertensive heart disease, a complex syndrome or a hypertensive 'cardiomyopathy'?" European Heart Journal:21 (20): 1653-65.

Living with Pulmonary Heart Disease, Retrieved (2010).

Lopez-Garcia, E., Schulze, M. B., Meigs, J. B., Manson, J. E., Rifai, N., Stampfer, M. J., Willett, W. C. and $\mathrm{Hu}, \mathrm{F}$. B. (2005): "Consumption of trans fatty acids is related to plasma biomarkers of inflammation and endothelial dysfunction". J Nutr:135 (3): 562-6.

Maeder, M. T. and Kaye, D. M. (2009): "Heart failure with normal left ventricular ejection fraction". Journal of the American College of Cardiology:53 (11): 905-918.

Maffuli, N., Chan, K., Macdonak, R., Malina, R.M. and Parker, A.W. (2003): Sports medicine for specific age group and abilities. Elservier health services Churchill Livingstone.

McCann, S. J. H. (2001): "The precocity-longevity hypothesis: earlier peaks in career achievement predict shorter lives". PersSocPsychol Bull:27: (11): 1429-39.

McKenzie, J., Neiger, B. and Thackeray, R. (2009): Health Education can also be seen as preventive medicine (2012): Health Education and Health Promotion. Planning, Implementing, \& Evaluating Health Promotion Programs: (pp. 3-4). 5th edition. San Francisco, CA: Pearson Education, Inc.

McWhinney, I. R. (1987): "Health and disease: problems of definition". CMAJ136 (8): 815.

Mitchell, R. S.,Kumar, V., Abbas, A. K. and Fausto, N. (2003): Robbins Basic Pathology (8 ${ }^{\text {th }}$ Ed.). Philadelphia: Saunders: ISBN 1-4160-2973-7.

Nattiv, A., \& Puffer, J.C. (1991): Lifestyles and health risks of collegiate athletes. TheJournal of Family Practice: 33 (6), 585-590.

Pieske, B. (2004): "Reverse remodeling in heart failure - fact or fiction?" Eur Heart J Suppl.6: D66-78. 


\section{Journal of Exercise Science \& Physiotherapy, Vol. 13, No. 2, 2017 \\ ISSN: 0973-2020 (Print) I I OR Impact Factor = 5.23 UGC Approved [Journal No.7485] ISSN: 2454-6089 (online)}

Prakash, D. (2014): Nuclear Medicine: A Guide for Healthcare Professionals and Patients: Springer Science \& Business Media p. 142: ISBN 9788132218265.

Robbins, S. L., Kumar, V. and Cotran, R. S. (2003): Robbins basic pathology ( $7^{\text {th }}$ Ed.). Philadelphia: Saunders: ISBN 0-7216-9274-5.

Ross, J. (2002): "Dilated cardiomyopathy: concepts derived from gene deficient and transgenic animal models". Circ J:66 (3): 219-24.

Ruiz-Canela, M. and Martínez-González, M. A. (2014): "Lifestyle and dietary risk factors for peripheral artery disease." Circulation journal: official journal of the Japanese Circulation Society78 (3): 553-9.

Selvin, E. and Erlinger, T. P. (2004): Prevalence of and risk factors for peripheral arterial disease in the united states results from the national health and nutrition examination survey, 1999-2000. Circulation: 110(6): 738-743.

Shanthi, M., Pekka, P. and Bo, N. (World Health Organization) (2011): Global Atlas on Cardiovascular Disease Prevention and Control (PDF). World Health Organization in collaboration with the World Heart Federation and the World Stroke Organization. pp. 3-18: ISBN 978-92-4-156437-3.

Stargrove, M. B., Treasure, J. and McKee, D. L. (2008): Herb, Nutrient, and Drug Interactions: Clinical Implications and Therapeutic Strategies: Elsevier Health Sciences: ISBN 0323029647 Retrieved (2015).

"Stroke: MedlinePlus Medical Encyclopedia" www.nlm.nih.gov: Retrieved (2015).

Swardfager, W., Herrmann, N., Cornish, S., Mazereeuw, G., Marzolini, S., Sham, L. and Lanctôt, K. L. (2012): "Exercise intervention and inflammatory markers in coronary artery disease: a metaanalysis". Am Heart J:163 (4): 666-676.

Taanila, H., Hemminki, A., Suni, J., Pihlajamäki, H. and Parkkari, J. (2011): Low physical fitness is a strong predictor of health problems among young men: a follow-up study of 1411 male conscripts. BMC Public Health; 11: 590.

Valentine, R. J., Guerra, R., Stephan, P., Scoggins, E., Clagett, G. P. and Cohen, J. (2004): "Family history is a major determinant of subclinical peripheral arterial disease in young adults". Journal of vascular surgery:39 (2): 351-356.

"Vascular dementia - Causes - NHS Choices" www.nhs.uk: Retrieved (2015).

Violi, F., Basili, S., Berger, J. S. and Hiatt, W. R. (2012): "Antiplatelet therapy in peripheral artery disease." Handbook of experimental pharmacology (210): 547-63.

Wang, X., Ouyang, Y., Liu, J., Zhu, M., Zhao, G., Bao, W. and Hu, F. B. (2014): "Fruit and vegetable consumption and mortality from all causes, cardiovascular disease, and cancer: systematic review and dose-response meta-analysis of prospective cohort studies." BMJ (Clinical research ed.)349: g4490.

Weiss, S. (1995): A comparison of maladaptive behaviors of athletes and non-athletes. Unpublished masters' Thesis-Springfield College

"What Are the Signs and Symptoms of Peripheral Arterial Disease?" (2011): http://www.nhlbi.nih.gov Retrieved (2015).

"What Is Peripheral Arterial Disease?" (2011): http://www.nhlbi.nih.gov Retrieved (2015).

"What Is Peripheral Vascular Disease?" (PDF) (2012):https://www.heart.org: Retrieved (2015).

Whitnet, E. and Rolfes, S. R. (2011): Understanding Nutrition. United States: Wadsworth Cengage Learning p. 255: ISBN 1-133-58752-6.

World Health Organization (1998): List of Basic Terms. Health Promotion Glossary: (pp. 4) Retrieved (2009).

World Health Organization (2002): Reducing risks,promoting healthy life: World Health Report 2002 Geneva, Switzerland. WHO Press

World Health Organization's (WHO's), (2010): global recommendations on: Physical Activity for Health. Geneva: World Health Organization.

World Health Organization (2008): The Global Burden of Disease: 2004 Update. Geneva: World Health Organization: ISBN 92-4-156371-0.

"WHO Disease and injury country estimates" World Health Organization (2009).

Xing, C., Arai, K., Lo, E. H. and Hommel, M. (2012): "Pathophysiologic cascades in ischemic stroke". International journal of stroke: official journal of the International Stroke Society:7 (5): 378-385.

Conflict of Interest: None Declared 